\title{
ROLE OF ANESTHESIOLOGISTS IN EARLY RECOGNITION OF TRAUMATIC INJURY OF THE THORACIC DUCT: CASE REPORT
}

\author{
Burmuzoska M. MD, Jovanovski - Srceva M. MD \\ 1PHI University Clinic Of Anesthesiology, Reanimation and Intensive care - Skopje
}

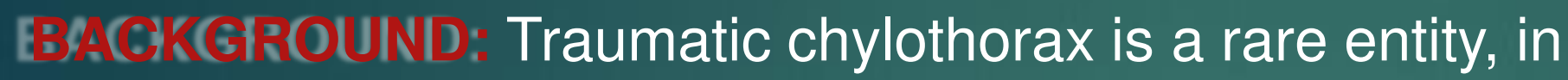
literature is known to be $2.6 \%$. Leak in the thoracic duct causes collection of chylous fluid in the pleural cavity, which can lead to alterations in pulmonary function and ventilation. Early recognition and good intensive care management followed by surgical treatment is crucial.

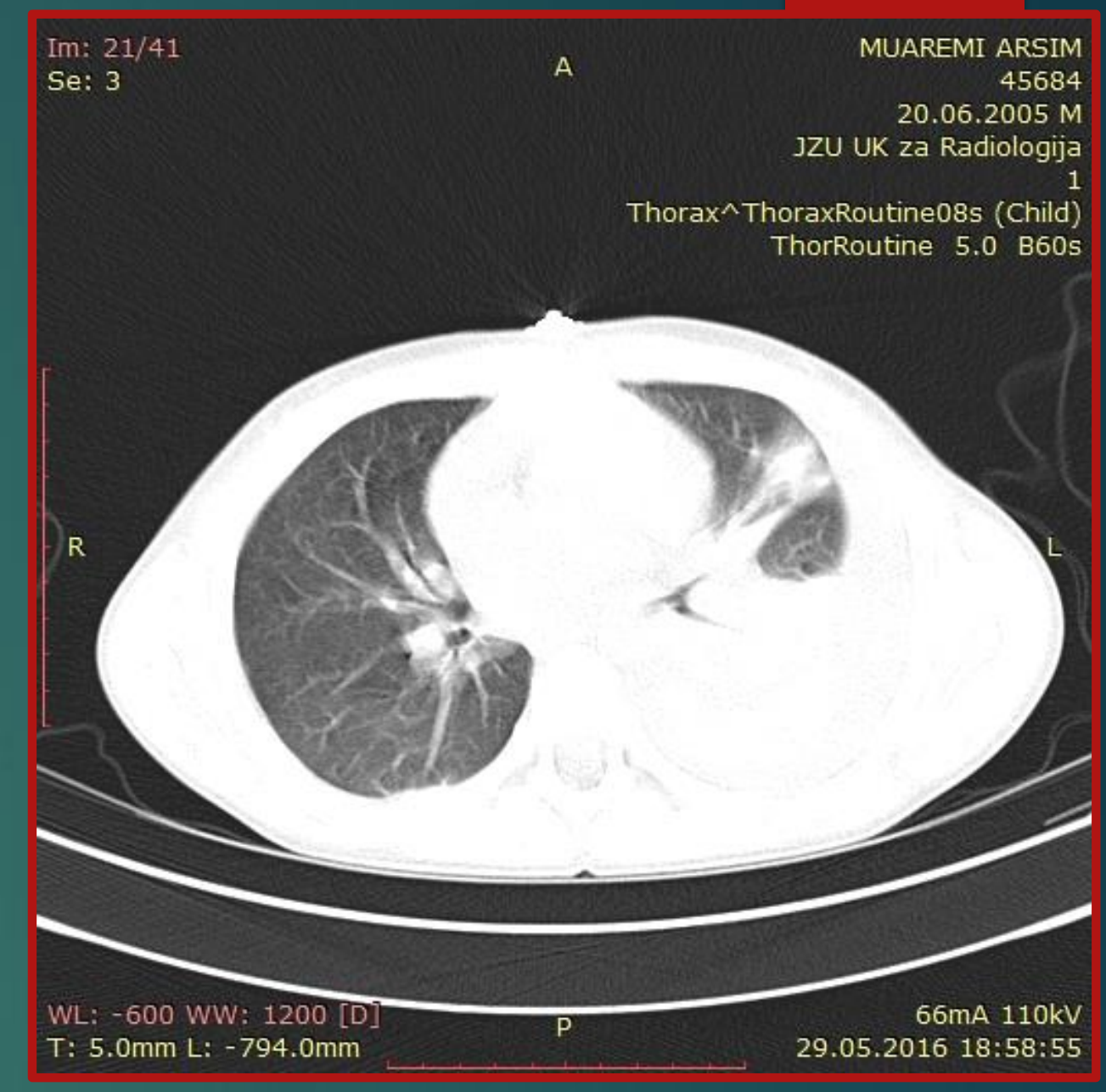

: An 11 years old boy was admitted to ICU after a stab wound in the left hemithorax. On physical examination, there was entrance wound, tachypnea and respiratory sounds were becoming lighter on the same side. After the initial CT scan, pleural effusion was seen and thoracic drainage was placed. Drainage of the effusion yielded a $2000 \mathrm{ml}$ cloudy, off-white fluid that settled in layers in the drainage container, in the first 24 hours. The drainage remained high in the next days and further investigations were made. Pleural fluid examination revealed a lymphocyte-rich transudate with high levels of cholesterol and triglycerides. All investigations indicated that there was lesion of the thoracic duct and indication for surgery was made.

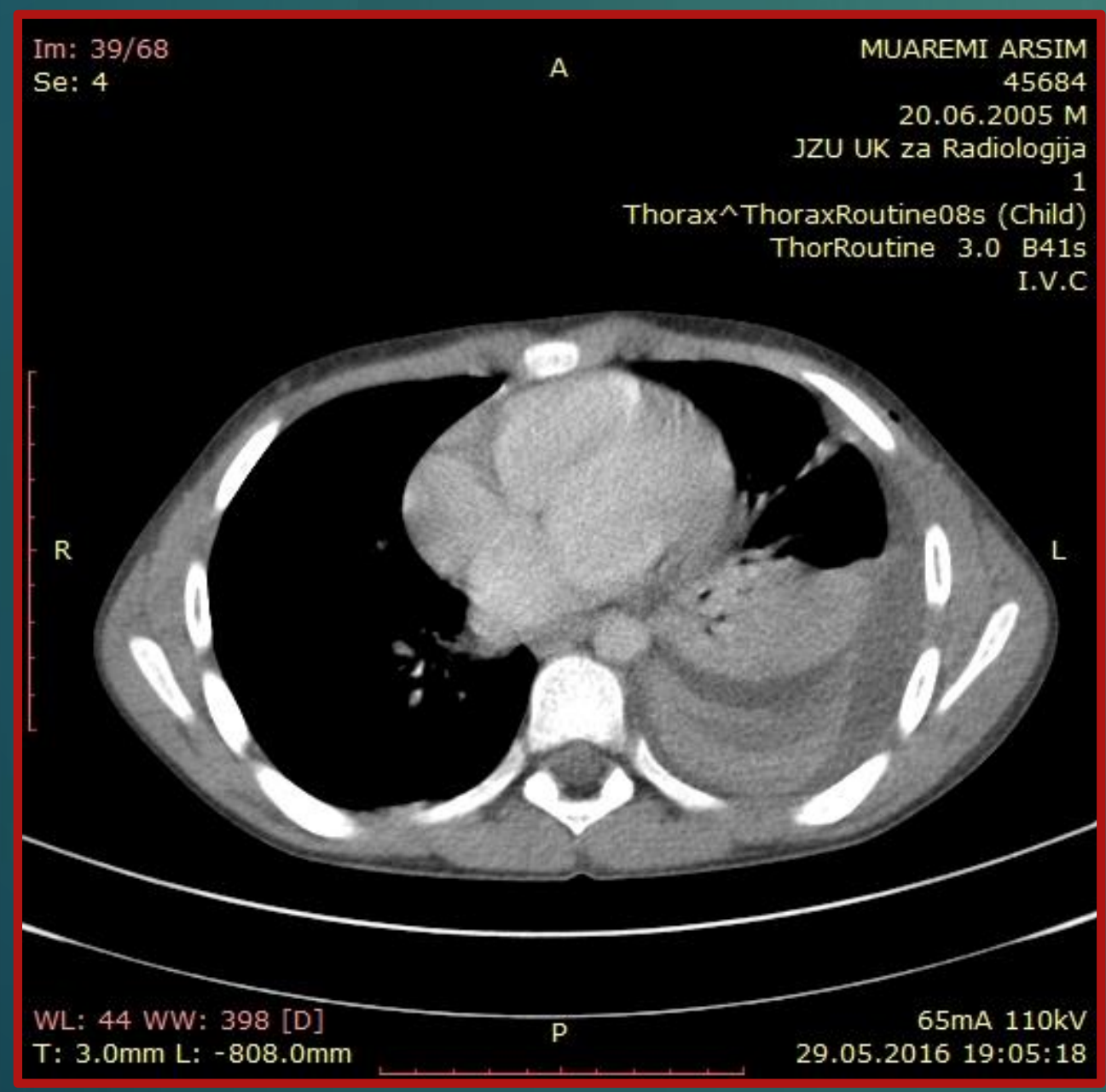

Traumatic chylothorax diagnosis is generally made on everyday evaluation on patient's condition and drainage fluid characteristics. Large amount of cloudy, offwhite fluid, which remains for several days, is a suspicion for anesthesiologist to analyze biochemically the content from thoracic drainage. Because of the large volume of lipid- and lymphocyte-rich fluid lost in a chyle leak, nutritional support of these patients is of paramount importance to prevent malnutrition, dehydration and immunosuppression. The timing for surgical treatment is controversial. Etiology and patient's overall condition are very important. 